\title{
Effet spécifique de trois virus sur l'expression de la résistance à une maladie cryptogamique du piment due à Phytophthora capsici Leon.
}

\author{
Edmond POCHARD $(*)$, Nadira CHALAL $\left({ }^{* *}\right)$ \& Georges MARCHOUX $\left({ }^{* *}\right)$ \\ (**) Institut national agronomique, El Harrach, DZ Alger (Algérie). \\ (*) I.N.R.A., Station d'Amélioration des Plantes maraîchères. \\ (***) I.N.R.A., Station de Pathologie végétale. \\ Centre de Recherches agronomiques d'Avignon, B.P. 94, F84140 Montfavet.
}

\begin{abstract}
RÉSUMÉ
L'infection préalable des plantes de piment (Capsicum annuum L.) par le virus de la mosaïque du tabac (TMV) supprime toute résistance à Phytophthora capsici Lcon. dans les tissus de la tige pendant la période de multiplication active du virus. L'infection par le virus Y de la pomme de terre (PVY) inhibe une phase tardive de cette même résistance. Le virus de la mosaïque du concombre (CMV) ne modifie pratiquement pas l'expression de la résistance à $P$. capsici.

Ces résultats semblent montrer que les 3 virus, qui sont des agents pathogènes fréquemment rencontrés sur Capsicum annuum L., modifient de façon spécifique le métabolisme cellulaire. Par ailleurs, on remarque que les mécanismes de résistance développés par le piment pour contrôler chacun de ces virus sont de type différent. Vis-à-vis de la mosaïque du tabac, on trouve un gène dominant induisant une hypersensibilité localisée. Les diverses résistances au virus $Y$ sont contrôlées par des gènes récessifs mettant en jeu des mécanismes de restriction de la multiplication. Il n'existe pas de résistance véritable au virus de la mosaïque du concombre mais des moindres sensibilités, de type polygénique, s'exprimant surtout au stade adulte. Il existe peut-être un lien entre le mode de multiplication de ces virus et la nature des résistances efficaces apparues sous la pression de la sélection naturelle.
\end{abstract}

\section{SUMMARY}

\section{Interaction}

virus-fungal disease,

Suppression of resistance,

$T M V$,

$P V Y$

$C M V$,

Phytophthora capsici,

Capsicum annuum
Specific effect of three viruses on the expression of resistance to a fungal disease of the bell pepper due to Phytophthora capsici Leon

Inoculation of pepper plants by tobacco mosaic virus (TMV) 8 to 18 days before Phytophthora capsici infection suppresses the resistance to the fungus in the stem tissues. Infection of the plants by potato virus $Y$ (PVY) inhibits a late phase of this resistance. Cucumber mosaic virus (CMV) has practically no effect on the expression of resistance to Phytophthora.

Those results seem to show that the three viruses, which are frequently found on Capsicum annuum, have a specific effect on the host cells metabolism. It is known that the resistance mechanisms developed during evolution by the pepper to control each of these viruses are different: dominant hypersensibility (TMV), recessive restriction of multiplication (PVY) or polygenic mature plant tolerance (CMV). It is suggested that a link could exist between the mode of multiplication of these viruses and the nature of the efficient resistance to the viral diseases.

\section{INTRODUCTION}

Le flétrissement du piment (Capsicum annuum L.) provoqué par Phytophthora capsici Leon. est une maladie redoutable des cultures irriguées méridionales, qu'elles soient conduites en serre ou en plein champ. Il est possible d'en ralentir la progression par la désinfection de l'eau d'arrosage ou par des traitements répétés au pied des plantes (MESSIAEN \& LAFON, 1970 ; CLERJEAU \& BEYRIES, 1977). Cependant l'efficacité de ces traitements n'est pas toujours assurée et la mise en cuvre en est coûteuse. La résistance génétique pourrait être la véritable solution à ce problème.

Jusqu'à une date récente, la meilleure source de résistance connue était constituée par une lignée à petit fruit originaire d'Amérique centrale (PI 201234 ), mise en évidence par KIMBLE \& GROGAN en 1960. Après vérification de la résistance dans les conditions françaises et autofécondations répétées, ce matériel a été enregistré dans notre collection sous le code PM 217. De nombreux travaux ont été conduits pour caractériser la nature et les modalités de cette résistance. Ainsi, on a pu montrer qu'il existait un 
mécanisme de défense induit par la présence du champignon et non préexistant à l'infection (PocHARD et al., 1976). Ce mécanisme met en jeu une séquence complexe d'événements parmi lesquels l'accumulation d'une phytoalexine : le capsidiol (Molot et al., 1976, 1980 ; Coulomb et al., 1979). Cependant, on note une grande hétérogénéité de réponse suivant les conditions de milieu, l'âge de la plante, la nature de l'organe inoculé ou l'agressivité de la souche employée (POCHARD et al., 1976; CleRJEAU et al., 1976).

La transmission héréditaire des propriétés de résistance n'est pas connue avec certitude. Aucune discontinuité n'apparaît dans les générations de disjonction entre résistants et sensibles dès lors qu'un test quantitatif précis est utilisé, mais la distribution est plurimodale en $F_{2}$. Les aptitudes d'inductibilité semblent contrôlées, au moins en partie, par 2 gènes complémentaires dominants (POCHARD \& ChambonneT, 1971) mais leur expression dépend du contexte génétique dans lequel ils sont introduits. On peut donc qualifier cette résistance de polygénique (ou d'oligopolygénique).

S'il n'est pas possible de déceler sans ambiguïté les déterminants héréditaires de la résistance, on peut tenter d'en analyser les composantes phénotypiques. D'après des études récentes (POCHARD \& DAUBEZE, 1980), ces composantes sont au nombre de 3 , intervenant successivement au cours du processus de l'infection. On a proposé de les désigner par les termes : « réceptivité », « inductibilité » et «stabilité de l'induction».

Dans les conditions réelles de culture, les plantes sont fréquemment attaquées par différents virus. Les principaux sont : le virus de la mosaïque du tabac ou TMV, répandu en serre, le virus Y de la pomme de terre ou PVY et le virus de la mosaïque du concombre ou CMV qui sévissent en plcin champ. Dans la région d'Avignon, la quasi-totalité des plantes se trouve, en fin de saison, infectée par ces 2 virus simultanément.

Le but des recherches entreprises est de voir si la présence de ces virus modifie la résistance à Phytophthora capsici. Si tel est le cas, il convient de révéler la nature et l'importance de ces effets: assiste-t-on à un renforcement ou, au contraire, à une inhibition de tout ou partie de la résistance à la maladic cryptogamique ?

\section{MATÉRIEL ET MÉTHODES}

\section{A. Matériel végétal}

Le matériel expérimental est constitué par 4 lignées homozygotes de piment, réparties en 2 paires partiellement isogéniques, sauf pour la résistance à $P$. capsici. La comparaison des 2 membres d'une paire permet de séparer les effets liés à la présence des gènes de résistance de ceux qui en sont indépendants (PocHARD \& DAUBEZE, 1980).

Ces lignées sont les suivantes:

— «Yolo Wonder» et «Phyo 636 » respectivement sensible et résistant à $P$. capsici. Cette paire est résistante au TMV et sensible aux 2 autres virus

- «Clairon» et « Fidélio », respectivement sensible et résistant à $P$. capsici. Cette paire est sensible aux 3 virus concernés.

Les plantes sont élevées en serre en godets de $10 \mathrm{~cm}$ sur un terreau enrichi en tourbe, jusqu'à l'apparition du $1^{\text {cr }}$ bouton floral.

\section{B. Matériel cryptogamique}

Les isolats utilisés sont ceux qui sont considérés comme les plus actifs dans l'induction du phénomène de résistance chez "Phyo 636»: S 15-12 A et S 101 (CLERJEAU et al., 1976). Ils sont cultivés pendant 8 à $10 \mathrm{j}$ sur le milieu $\mathrm{L}$ de MESSIAEN \& LAFON (1970).

\section{Matériel viral}

Virus de la mosaïque du tabac (TMV): L'inoculum est constitué par un extrait aqueux (dilué au 1/40 $)$ de feuille de piment infecté depuis 2 semaines par la souche Vi-76 (isolée sur piment, elle appartient au groupe «tabac»). Cette souche donne une mosaïque franche sur les génotypes sensibles, les symptômes commençant à apparaître 8 à $10 \mathrm{j}$ après l'inoculation. Chez les variétés résistantes portant le gène $L^{i}$ (HoLmes, 1937) elle provoque, en 3 à $5 \mathrm{j}$, la formation de lésions locales sur les feuilles inoculées qui ne tardent pas à jaunir et à tomber. Par la suite, les plantes sont indemnes de virus.

Virus $Y$ de la pomme de terre $(P V Y)$ : La souche utilisée porte le code N 17-D. Elle attaque les 4 variétés à l'essai, donnant une mosaïque nervaire classique sur les jeunes feuilles 7 à $9 \mathrm{j}$ après la contamination d'une feuille adulte. Les méthodes d'extraction et d'inoculation ont été précédemment décrites (PochaRd, 1977).

Virus de la mosaïque du concombre (CMV) : L'inoculum est obtenu à partir de la souche TL représentative des populations virales les plus répandues, en plein été, dans la région d'Avignon (QuIOT et al., 1979). L'extraction est faite à froid en présence de charbon végétal suivant la méthode de MARROU (1967), à raison de $1 \mathrm{~g}$ de feuille infectée pour $4 \mathrm{ml}$ de solution. La plante donneuse appartient à la variété «Yolo Wonder » inoculée 2 semaines auparavant.

\section{Préinoculation par un virus}

L'infection artificielle est effectuée sur la $6^{\mathrm{e}}$ feuille, alors que la plante possède au moins 8 feuilles étalées. La feuille est saupoudrée de carborundum 400 mesh et inoculéc par frottis. Au bout de $2 \mathrm{mn}$ environ, la feuille est lavée pour éliminer les restes de milieu d'extraction. Les plantes témoins sont traitées de la même façon mais à partir de broyats de feuilles saines.

L'intervalle entre l'inoculation virale et l'inoculation par le champignon est de 7 ou $8 \mathrm{j}$ (début d'apparition des symptômes viraux sur les jeunes feuilles) ou 15 à $18 \mathrm{j}$ (symptômes bien établis sur l'ensemble de la plante).

\section{E. Inoculation et notation de Phytophthora}

Les plantes sont placées en chambre climatisée à $22^{\circ} \mathrm{C}$, sous un éclairage mixte donnant environ $50 \mathrm{~W} . \mathrm{m}^{-2}$. Les plantes sont décapitées au-dessous de la $8^{\mathrm{c}}$ feuille et l'on dépose immédiatement sur la section un disque de culture pure du champignon d'un diamètre comparable à celui de la tige (4 à $5 \mathrm{~mm}$ ). La zone ainsi infestée est entourée d'un papier d'aluminium formant chambre humide sur une longueur d'environ $2 \mathrm{~cm}$.

La notation consiste en la mesure, au mm près, de la longueur de la nécrose induite par le champignon. Cette notation commence 2 ou $3 \mathrm{j}$ après l'inoculation et se poursuit pendant 2 semaines à intervalles de 3 ou $4 \mathrm{j}$. La vitesse moyenne de progression de la nécrose, exprimée en $\mathrm{mm}$ par jour, est rapportée au dernier jour de la période 
correspondante. Le nombre de plantes par traitement élémentaire est en général 10. Les échantillons témoins et préinoculés sont disposés en lignes parallèles et la comparaison statistique est effectuée au moyen du test $\mathbf{t}$ de Student.

Chaque type d'essai a été effectué au moins 2 fois.

\section{RÉSULTATS}

L'existence d'un effet d'interférence entre les 2 agents pathogènes est décelée grâce à la comparaison des vitesses de nécrose de la tige chez les plantes témoins qui n'ont reçu

\section{Vitesse de nécrose}

$\left(\mathrm{mm} . \mathrm{j}^{-1}\right)$
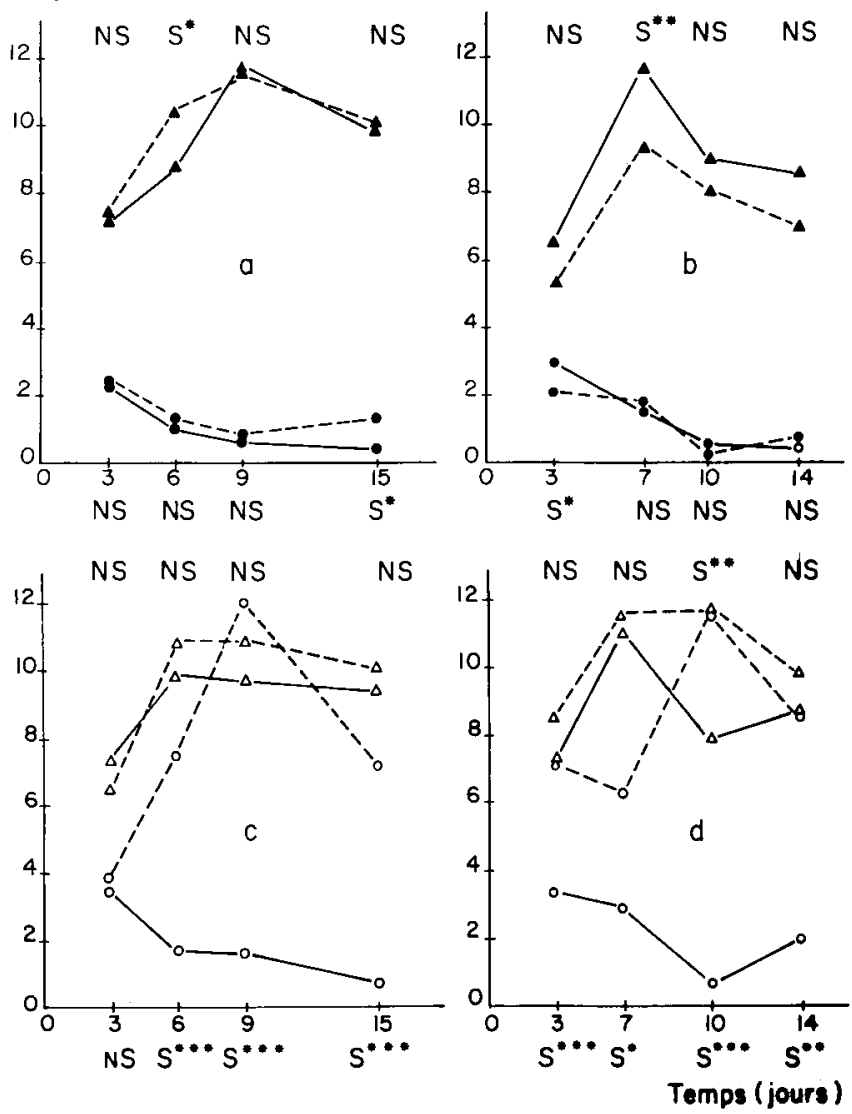

Figure 1

Effet d'une préinoculation par le virus de la mosaïque du tabac (TMV) sur la vitesse de nécrose de la tige de piment induite par Phytophthora capsici.

NS: effet non significatif; $S$ : effet significatif au seuil $0,05\left(^{*}\right)$, 0,01 (**) $^{*} 0,001$ (***). $^{*}$.

Témoin non préinoculé : trait continu; préinoculé par le TMV : trait interrompu.

Variétés résistantes au P. capsici : "Phyo 636 (О); "Fidélio $\gg(\mathrm{O})$.

Variétés sensibles au P. capsici : "Yolo Wonder" (A); "Clairon"

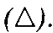

$1 a$ et $1 b$ : paire résistante au $\operatorname{TMV}(\mathbf{O}, \mathbf{\Delta}) ; 1$ c et $1 d$ : paire sensible au $T M V(O, \triangle)$.

Temps séparant les 2 inoculations : $8 j(1 a, 1 c) ; 18 j(1 b, 1 d)$.

Effect of a preinoculation by tobacco mosaic virus (TMV) on the speed of stem necrosis induced by Phytophthora capsici.

$N S$ : no significative effect; $S$ : significative effect at the level $0,05\left(^{*}\right), 0,01\left(^{* *}\right), 0,001\left(^{* * *}\right)$.

Control without viral infection : full line; preinoculated by TMV: dotted line.

Varieties resistant to P. capsici : Phyo $636(0)$; Fidelio (O).

Varieties susceptible to P. capsici : Yolo Wonder (A); Clairon $(\Delta)$. $1 a$ and $1 b$ : near isogenic pair resistant to $T M V(\mathbf{0}, \mathbf{\Delta})$; susceptible to $T M V(O, \triangle)$; interval between the successive inoculations: 8 days $(1 a, 1 d) ; 18$ days $(1 b, 1 d)$. que le Phytophthora et chez celles qui ont été tout d'abord inoculées par un virus. Les mesures étant non destructives, on peut les répéter plusieurs fois et apprécier le moment où cet effet se produit au cours du développement de l'infection, chez les variétés sensibles comme chez les variétés résistantes au champignon. La vitesse de nécrose donne une image fidèle de la rapidité de progression du champignon dans les tissus de la tige (MOLOT et al., 1976).

\section{A. Interférence mosaïque du tabac Phytophthora}

Chez les 2 variétés résistantes au virus de la mosaïque du tabac, la préinoculation par ce virus n'a pas d'effet important sur le déroulement de l'infection par Phytophthora. L'effet est nul chez «Phyo 636" (partenaire résistant). Chez le partenaire non résistant à $P$. capsici, "Yolo Wonder », on note une légère diminution de la vitesse de nécrose de la tige lorsque $18 \mathrm{j}$ séparent les 2 inoculations (fig. $1 a$ et $1 b$ ).

Les résultats sont tout autres lorsque l'on se tourne vers les 2 variétés sensibles au TMV. On constate ici que la présence du virus entraîne une suppression quasi totale de la résistance au champignon chez «Fidélio ». La résistance à $P$. capsici s'exprime normalement par une forte décroissance de la vitesse de nécrose de la tige entre le $3^{\text {e }}$ et le $9^{\mathrm{e}}$ jour. Chez les plantes préinoculées par le TMV, cette vitesse augmente durant la même période, comme si la résistance ne pouvait s'exprimer ou était très fortement inhibće. Chez le partenaire «Clairon», sensible au Phytophthora, on observe une légère augmentation de la vitesse de nécrose de la tige si le virus est inoculé $18 \mathrm{j}$ avant le champignon (fig. $1 c$ et $1 d$ ).

\section{B. Interférence virus Y de la pomme de terre Phytoph- thora}

Chez « Yolo Wonder » et «Clairon», on remarque que le comportement vis-à-vis de Phytophthora n'est pas modifié par la présence du virus.

Par contre, on note l'existence d'effets très significatifs chez «Phyo 636 » et «Fidélio», partenaires résistants au Phytophthora. Ces effets s'expriment le mieux lorsque 7 jours séparent les 2 inoculations; ils s'atténuent lorsquc cet intervalle est plus long.

Dans le $1^{\text {cr }}$ cas, le déroulement de la résistance apparaît tout d'abord normal : entre le début de l'infection et le $6^{\mathrm{e}} \mathrm{j}$, la résistance se déclenche de la même façon chez les témoins et chez les plantes préinoculécs par lc PVY. Par contre, entre le $6^{\mathrm{e}}$ et le $15^{\mathrm{e}} \mathrm{j}$, la vitesse de nécrose est significativement plus grande chez les plantes virosées (fig. $2 a$ et $2 c$ ).

Lorsque le champignon est apporté $15 \mathrm{j}$ après le virus, l'effet est atténué chez «Phyo 636» et indiscernable chez "Fidélio " (fig. $2 b$ et $2 d$ ). L'effet paraît donc beaucoup moins persistant que celui qui est provoqué par le TMV.

\section{Interférence virus de la mosaïque du concombre Phytophthora}

Que l'intervalle séparant les 2 inoculations soit court (8 j) ou long (18 j), on n'observe aucune différence majeure de comportement entre les plantes virosées et les plantes témoins. La figure 3 montre que les courbes d'évolution de la vitesse de nécrose sont pratiquement confondues. Tout au plus note-t-on une légère augmentation de la vitesse initiale de pénétration du champignon sur les plantes contaminées depuis $8 \mathrm{j}$ par le CMV, mais cet effet n'apparaît pas régulièrement dans tous les cssais. 


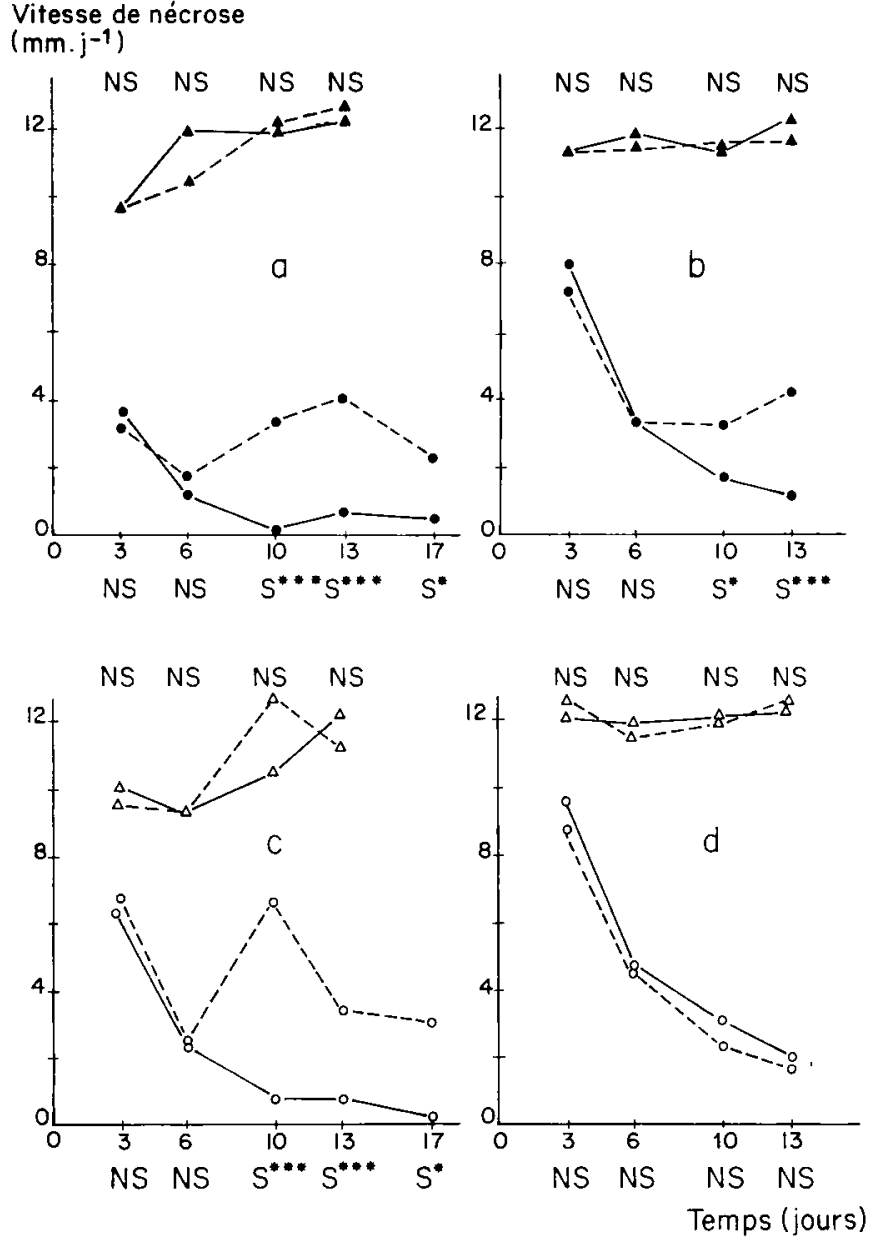

Figure 2

Effet d'une préinoculation par le virus $Y$ de la pomme de terre $(P \vee Y)$.

Mêmes variétés et même disposition que sur la figure 1.

Les 4 variétés sont sensibles au PVY; temps séparant les 2 inoculations: $7 j(2 a, 2 c) ; 15 j(2 b, 2 d)$.

Effect of a preinoculation by potato virus $Y(P V Y)$ on the speed of stem necrosis induced by Phytophthora capsici.

Same varieties and same arrangement as in figure 1.

The 4 varieties are susceptible to $P V Y$; interval between 2 inoculations: 7 days $(2 a, 2 c) ; 15$ days $(2 b, 2 d)$.

\section{DISCUSSION}

Il convient de distinguer les effets mineurs de faible amplitude, n'apparaissant que dans certains essais ou sur certaines variétés, des effets majeurs qui s'expriment systématiquement dans tous les essais et changent complètement le déroulement de la relation entre la plante et le champignon pathogène.

Nous ne nous attarderons pas sur les premiers. L'induction d'une résistance partielle à $P$. capsici chez un génotype sensible à cet agent par une inoculation virale incompatible n'a pu être démontrée. La réduction de sensibilité, quand elle existe («Yolo Wonder » préinoculé par le TMV depuis $18 \mathrm{j}$, fig. $1 b$ ) est peu importante et ne change pas le résultat final qui est la mort de la plante. Des essais similaires, conduits avec d'autres virus incompatibles donnant des lésions locales, n'ont pas donné de réponses plus favorables (données non publiées).

Les effets majeurs concernent tous la suppression partielle ou totale de la résistance au Phytophthora. Cependant, les 3 virus TMV, PVY et CMV donnent des effets différents. Le virus de la mosaïque du tabac a la propriété

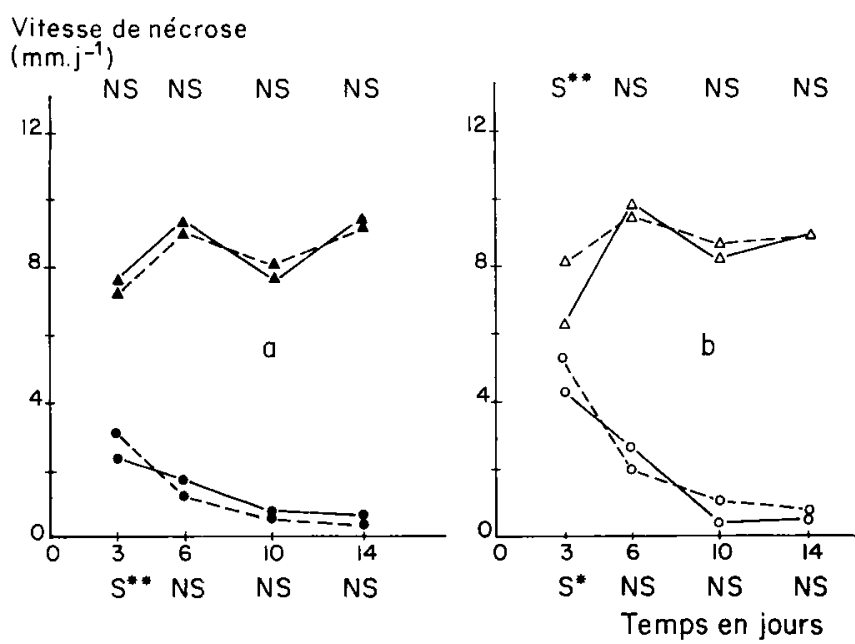

Figure 3

Effet d'une préinoculation par le virus de la mosaïque du concombre (CMV).

Mêmes variétés que figures 1 et 2 .

Ces 4 variétés sont sensibles au CMV; intervalle entre les 2 inoculations : 8 j: $3 a$ : "Yolo Wonder " et "Phyo 636 "; $3 b:$ "Clairon" et "Fidélio".

Effect of a preinoculation by cucumber mosaic virus (CMV). Same varieties as in figures 1 and 2.

The 4 varieties are susceptible to CMV; interval between the 2 inoculations : 8 days : $3 a$ : "Yolo Wonder" and "Phyo 636 "; $3 b$ : "Clairon " and "Fidélio ".

d'empêcher l'expression de la résistance à $P$. capsici pendant la totalité de la période étudiée. Pratiquement, la résistance est supprimée puisque le déroulement de l'infection chez la lignée génétiquement résistante ne se distingue plus de celui de la lignée sœur sensible ne portant pas de gènes de résistance au Phytophthora (couple quasi isogénique « Fidélio » et « Clairon»).

Le cas du PVY est différent du précédent puisque l'effet d'inhibition de la résistance est localisé dans le temps et l'espace. Ce virus ne modifie pas le déroulement des premières étapes de la résistance à $P$. capsici. Par contre, il provoque une reprise de croissance active du champignon dans la tige du piment au cours de la $2^{\mathrm{e}}$ phase de l'infection, soit 6 jours environ après la contamination. Cet effet n'est observable que si le virus a envahi les tissus de la tige depuis peu de temps. Trois semaines après l'inoculation virale, il est déjà très atténué ou inexistant suivant la variété considérée.

Le virus de la mosaïque du concombre, qui n'est pas un virus moins dangereux que les 2 précédents dans les conditions naturelles, montre une discrétion exemplaire. On n'a pas pu déceler d'effet lié à sa présence quel que soit l'intervalle de temps séparant les 2 inoculations.

La littérature nous offre d'assez nombreux exemples de sensibilisation à une maladie cryptogamique provoquée par la présence d'un virus compatible. Ainsi, chez les variétés de concombre infectées par le CMV, la surinfection par Pythium ultimum entraîne un flétrissement qui n'est pas observé chez les variétés résistantes à ce virus (NITZANI, 1966). Chez la pomme de terre, les infections au champ par Phytophthora infestans apparaissent plus tôt et sont plus intenses sur les plantes déjà atteintes par le virus de l'enroulement que chez les plantes apparemment saines (Novakova, 1977). Sous l'effet du virus, une variété résistante peut se trouver déclassée. 
Chez la betterave à sucre, lcs interactions virus-champignon jouent un rôle considérable dans l'épidémiologie. Ainsi, l'Alternaria est rarement présent dans les cultures saines ou atteintes de jaunisse à beet yellows virus (ou BYV) mais il peut provoquer des pertes sévères chez les plantes atteintes de beet mild yellowing virus (ou BMYV) (RUSSELL, 1966a). L'effet de ces 2 virus est encore plus contrasté si l'on considère la vulnérabilité aux attaques d'Erysiphe betae : le BMYV rend les feuilles plus sensibles à l'ö̈dium alors que le BYV les rend plus résistantes (RUSSELL, 1966c).

Nous trouvons donc chez la betterave un effet différent suivant le virus infecticux. Au sein d'un même virus, les diverses souches peuvent se comporter différemment et les conditions de milieu peuvent aussi contrôler l'interaction. La résistance «non spécifique » à Peronospora farinosa est diminuée, en plein champ, par certains isolats de BYV et de BMYV. Cependant, d'autres isolats n'ont pas d'action décelable et d'autres encore augmentent la résistance (RUSSELL, 1966b). En inoculation artificielle sur de jeunes plantes, les effets, quand il y en a, vont tous dans le sens d'une augmentation de la résistance (RUSSELL, 1969). Si l'on pulvérise du saccharose ou du glucose sur les feuilles, on augmente leur résistance au Peronospora. A l'inverse, des traitements préalables d'ombrage ou de défoliation partielle (supposés diminuer la quantité de sucres disponible dans les feuilles) affaiblissent cette résistance (RUSSELL, 1968).

La versatilité des effets observés est attribuée par RusSELL (1970) au fait qu'il s'agit de résistances polygéniques n'impliquant pas l'hypersensibilité mais liées au métabolisme cellulaire général. La concentration en sucres dans les tissus pourrait jouer un rôle important.

Nos résultats ne semblent pas pouvoir être expliqués par de simples différences quantitatives facilement réversibles.

Tout d'abord, nous étudions une résistance induite qui met très vraisemblablement en œuvre des mécanismes d'hypersensibilité (nécrose des tissus, apparition de substances spécifiques, suppression de la résistance par les hautes températures, présence d'éliciteurs de résistance dans les filtrats de culture de champignon, etc... (MoLot et al., 1976, 1980).

En second lieu, la résistance du piment à $P$. capsici ne peut pas être décrite comme non spécifique : il existe des interactions significatives variétés-souches (POCHARD \& DAUBEzE, 1980). Elle peut être considérée comme polygénique mais cela n'exclut pas qu'il puisse exister un ou plusieurs gènes majeurs contrôlant l'hypersensibilité (Pochard \& Chambonnet, 1971).

Enfin, s'il y avait un effet de prédisposition à la maladie indépendant de la présence des gènes de résistance, on le retrouverait chez le partenaire sensible des couples quasi isogéniques, ce qui n'est pas le cas.

On peut donc avancer l'hypothèse d'une répression spécifique de certains éléments de la résistance à Phytophthora par le TMV ou le PVY en cours de multiplication, le CMV ne modifiant pas le déroulement de la résistance. Il serait hasardeux de spéculer sur la nature de ces effets mais on est amené à s'interroger sur les particularités biologiques des 3 virus à l'essai qui pourraient rendre compte du mode d'interaction particulier à chacun d'entre eux.

La stabilité in vivo appréciée par le pouvoir pathogène des extraits varie suivant les virus considérés. Si le TMV et le PVY sont stables (GoODschild et al., 1958 ; SIMONS, 1958), il n'en va pas de même du CMV (MARROU et al., 1968). La quantité de nucléoprotéines purifiables et leur activité infectieuse spécifique diminuent rapidement dans les feuilles infectées (MARCHOUX et al., 1975).

On sait aussi que chacun des virus conduit à la formation d'inclusions caractéristiques (CHRISTIE \& EDWARDSON, 1977), paracristaux, «pinwheels » ou inclusions vacuolées, respectivement, qui traduisent une modification du fonctionnement cellulaire de l'hôte dépendant du virus infectieux.

Ces éléments ne sont pas suffisants pour mettre sur la voix d'une interprétation de l'effet spécifique d'interaction.

Si l'on considère les mécanismes de résistance que le piment a développés pour contrôler chacun des 3 virus, on trouve une situation très contrastée.

La résistance au TMV est assez commune chez Capsicum annuum ; elle est la règle chez d'autres espèces de Capsicum (C. frutescens, C. chinense, C. baccatum). Dans tous les cas, il s'agit d'une résistance par hypersensibilité localisée, contrôlée par des gènes dominants appartenant à une série allélique (Holmes, 1937 ; POCHARD, 1965 ; BOUKEMA, 1980). On ne connaît pas d'autres formes de résistance.

La résistance au PVY est d'un type tout différent. Liée à des gènes récessifs, elle ne met pas en jeu d'hypersensibilité (COOK, 1960a; PAHLEN et al., 1973; POCHARD, $1977 b$ ). Elle est parfois qualifiée d'immunité mais ce terme semble impropre car il y a une faible multiplication au sein de la feuille inoculée, suffisante pour empêcher, pendant quelques jours, la multiplication d'une souche compatible du même virus (données non publiées). Cette résistance s'exprime mal dans les conditions hivernales (SHIFRISS \& COHEN, 1971). Les sources sont nombreuses. Une mutation de résistance a été décelée par CoOK (1960b) au sein d'une variété sensible.

On ne connaît pas de véritable résistance au CMV au sein du genre Capsicum mais une série de moindres sensibilités au stade adulte, d'hérédité polygénique (PocHARD, 1977a).

Ainsi, les 3 grands types de résistance aux virus se trouvent présents chez le piment. Vis-à-vis du TMV, on trouve donc l'hypersensibilité. Il se pourrait que la sensibilité au TMV implique la répression du mécanisme d'hypersensibilité et cette répression pourrait s'étendre à d'autres agents pathogènes, eux-mêmes inducteurs de ce mécanisme (cas de $P$. capsici). Si les mécanismes de reconnaissance à l'origine de la résistance sont certainement spécifiques de l'agent pathogène, le déroulement de l'hypersensibilité pourrait comporter des séquences communes, d'où l'effet d'interférence.

Le PVY n'est pas le seul agent capable de provoquer une instabilité à un moment précis du déroulement de la relation de résistance. Certaines souches de Phytophthora ont une tendance à induire spontanément cette même rupture de résistance. Certains génotypes présentent ce phénomène plus fréquemment que d'autres, particulièrement chez les plantes âgées (Pochard \& DAUBEzE, 1980 et données non publiées). On peut supposer que la résistance comporte l'action en chaîne de 2 processus distincts. Le PVY interférerait directement avec le second, laissant le premier inchangé.

Le CMV, enfin, ne perturbe aucun de ces mécanismes. Peut-être n'a-t-il pas besoin, pour assurer sa propre multiplication, de réprimer les défenses de l'hôte. Est-ce cette discrétion qui lui permet de ne pas susciter l'apparition de mutations de résistance, alors que cela s'est produit dans le cas du PVY et sans doute de nombreuses fois puisqu'il existe de nombreuses sources indépendantes? 
Ces ćtudes comportent des conséquences pratiques. Pour assurer une bonne protection des cultures de piment contre $P$. capsici, il convient d'associer aux gènes qui contrôlent la résistance à ce champignon, ceux qui conditionnent la résistance aux souches prévalentes des 2 virus, TMV et PVY. Des travaux dans ce sens sont en cours chez plusieurs établissements de sélection français et étrangers.

Reçu le 29 décembre 1980 Accepté le 20 mars 1981

\section{REMERCIEMENTS}

Les autcurs remercient Augustine FLORENT et Anne-Maric DAUBEZE pour leur aide efficace dans la réalisation des essais. Ils sont reconnaissants à J. Ponchet (I.N.R.A., Antibes) et C. M. MEssiaen (I.N.R.A., Montpellier) d'avoir aidé à la mise en place définitive du texte de l'article grâce à leurs commentaires constructifs.

\section{RÉFÉRENCES BIBLIOGRAPHIQUES}

Boukema I. W., 1980. Allelism of genes controlling resistance to TMV in Capsicum L. Euphytica, 29 (2), 433-439.

Christie R. G., Edwardson J. R., 1977. Light and electron microscopy of plant virus inclusions. Fla. Agric. Exp. Stn., Monogr. $\mathrm{n}^{\circ} 9,150 \mathrm{p}$.

Clerjeau M., Beyries A., 1977. Etude comparée de l'action préventive et du pouvoir systémique de quelques fongicides nouveaux (phosphites, prothiocarbe, pyroxychlore) sur poivron vis-à-vis de Phytopthora capsici Leon. Phytiatr. Phytopharm., 26, 73-84.

Clerjeau M., Nourrisseau J. G., 1976. La résistance du piment (Capsicum annuum) à Phytophthora capsici. II. Variation de la réceptivité des tissus voisins de la zone infectée induite par la contamination. Ann. Phytopathol., 8 (4), 389-397.

Cook A. A., 1960a. Genetics of resistance in Capsicum annuum to two virus discascs. Phytopathology, 50, 364-367.

Cook A. A., 1960b. A mutation for resistance to potato virus $Y$ in pepper. Phytopathology (Abst.), 50, 632.

Coulomb P. J., Molot P. M., Mas P., Conus M., Ferrière H., 1980. Mise en évidence d'unc induction de capsidiol et de résistance chez Capsicum annuum par certaincs fractions cellulaires obtenues après fractionnement du mycélium de Phytophthora capsici. C.R. Acad. Sci., Paris Série D, 290 (3), 275-277.

Goodschild D. J., Cohen M., Wildman S. G., 1958. The specific activity of tobacco mosaic virus as a function of age of infection. Virology, 5, 561-566.

Holmes F. O., 1937. Inheritance of resistance to tobacco mosaic disease in the pepper. Phytopathology, 27, 637-642.

Kimble K. A., Grogan R. G., 1960. Resistance to Phytophthora root rot in pepper. Plant Dis. Rep., 44 (11), 872-873.

Marchoux G., Douine L., Esvan Ch., 1975. Etude préliminaire sur la variation de l'activité infecticuse du virus de la mosaíque du concombre. Meded. Fac. Landbouw Rijks. Univ. Gent, 40, 73-88.

Marrou J., 1967. Amćlioration des méthodes de transmission mécanique des virus par adsorption des inhibitcurs d'infection sur lc charbon végétal. C.R. Acad. Agric. Fr., 55, 972-981.

Marrou, J., Migliori A., Marchoux G., 1968. Variation dans le temps du pouvoir infectieux du virus de la mosaique du concombre chez différentes espèces végétales. Etudes de virologie. Ann. Epiphyt., 19 (H.S.), 83-92.

Messiaen C. M., Lafon R., 1970. Les maladies des plantes maraîchères. I.N.R.A. Paris, $2^{c}$ ćd. 387-388.

Molot P. M., Clerjeau M., Nourrisseau J. G., Ricci P., 1976. La résistance du pimcnt (Capsicum annuum) à Phytophthora capsici Leon. III. Etude, sur extraits de tiges sensibles et résistantes, du pouvoir antifongique induit par la contamination. Ann. Phytopathol., 8 (4), 399-409.

Molot P. M., Mas P., Hilario A. L., 1980. La résistance du piment (Capsicum annuum) à Phytophthora capsici. VI. Pouvoir inhibitcur du capsidiol sur la croissance et l'activité pectinolytique d'isolats d'agressivité variable. Ann. Phytopathol., 12 (1), 1-9.

Molot P. M., Mas P., Conus M., Ferrière H., 1980. La résistance du piment à Phytophthora capsici. VII. Protection des organcs foliaires après mise en survie sur un filtrat de culture du parasite: caractéristiques des conditions d'expression du phénomène. Ann. Phytopathol., 12 (2), 95-107.

Nitzany F. E., 1966. Synergism between Pythium ultimum and cucumber mosaic virus. Phytopathology, 56, 1386-1389.
Novakova J., 1977. Interaction between leaf roll virus and late blight in potatoes (tch.-an) Ochrana Rostlin, 13, 201-209.

Pahlen A. (von der), Nagai H., 1973. Resistencia del pimiento (Capsicum sp.) a estirpes predominantes del virus $\mathrm{Y}$ de la papa en Bucnos Aires, el N.O. argentino y en el centro sur del Brasil. Rev. Invest. Agropecu., I.N.T.A., Pathol. Veg., 10, 109-116.

Pochard E., 1977a. Méthodes pour l'étude de la résistance partielle au virus du concombre chez le piment. "Capsicum 77 », C.R. $3^{e}$ Congrès Eucarpia, 5-8 juil. Edit. E. POCHARD, I.N.R.A. 84140 Montfavet-Avignon, France, 93-104.

Pochard E., 1977b. Etude de la résistance aux souches européennes de virus Y de la pomme de terre (PVY) chez le piment. "Capsicum 77 ", C.R. $3^{e}$ Congrès Eucarpia, 5-8 juil. Edit. E. POCHARD, I.N.R.A. 84140 Montfavet-Avignon, France, 109-118.

Pochard E., Breuils G., 1965. La résistance du piment (Capsicum annuum L.) à la mosaïque du tabac et au virus 1 du concombre. Modalités et transmission héréditaire. $1^{r e}$ Journ. Phytiatr. Phyto pharm., Marseille, 189-193.

Pochard E., Chambonnet D., 1971. Méthodes de sélection du piment pour la résistance au Phytophthora capsici et au virus du concombre. Eucarpia Meeting on Capsicum, Torino, 1971. Ann. Fac. Sci. Agr. Univ. Torino, 7, 270-281.

Pochard E., Clerjeau M., Pitrat M., 1976. La résistance du piment, Capsicum annuum L. à Phytophthora capsici Lcon. I. Mise en évidence d'une induction progressive de la résistance. Ann. Amélior. Plantes, 26 (1), 35-50.

Pochard E., Daubeze A. M., 1980. Rechcrche et évaluation des composantes d'une résistance polygénique : la résistance du piment à Phytophthora capsici. Ann. Amélior. Plantes, 30 (4), 377-397.

Quiot J. B., Devergne J. C., Marchoux G., Cardin L., Douine L., 1979. Ecologie et ćpidémiologie du virus de la mosaique du concombre dans le Sud-Est de la France. VI. Conservation de 2 types de populations virales dans les plantes sauvages. Ann. Phytopathol., 11 (3), 349-357.

Russell G. E., 1966a. The control of Alternaria species on leaves of sugar beet infected with yellowing viruses. II. Expcriments with two yellowing viruses and virus-tolerant sugar beet. Ann. appl. Biol., 57, 425-434.

Russell G. E., 1966b. Some effects of inoculation with yellowing viruses on the susceptibility of sugar beet to fungal pathogens. I. Susceptibility to Erysiphe species. Trans. Brit. Mycol. Soc., 49, 621628.

Russell G. E., 1966c. Some effects of inoculation with yellowing viruses on the susceptibility of sugar beet to fungal parthogens. II Susccptibility to Erysiphe species. Trans. Brit. Mycol. Soc., 49, 621-628.

Russell G. E., 1968. The influence of foliar applications of sugars on the susceptibility of sugar bect to downy mildew. Ann. appl. Biol., 61, 381-386.

Russell G. E., 1969. Specific interactions between virus and fungus diseases in sugar beet. J. Inst. intern. Rec. better, 3, 176-181.

Russell G. E., 1970. Interactions between diseases of sugar bect leaves. N.A.A.S.Q. Rev., 87, 132-138.

Shifriss C., Cohen S., 1971. Environmental modification of heritable resistance to potato virus $\mathrm{Y}$ in peppers, Capsicum annuum. Plant. Dis. Rep., 55, 604-606.

Simons J. N., 1958. Titers of three nonpersistent aphid-borne viruses affecting pepper in South Florida. Phytopathology, 48, 265 268. 